PROCEEDINGS OF THE

AMERICAN MATHEMATICAL SOCIETY

Volume 126, Number 8, August 1998, Pages 2323-2331

S $0002-9939(98) 04272-5$

\title{
CONVEX LINEAR COMBINATIONS OF SEQUENCES OF MONIC ORTHOGONAL POLYNOMIALS
}

\author{
A. CACHAFEIRO AND F. MARCELLAN
}

(Communicated by J. Marshall Ash)

\begin{abstract}
For a sequence $\left\{\Phi_{n}\right\}_{0}^{\infty}$ of monic orthogonal polynomials (SMOP), with respect to a positive measure supported on the unit circle, we obtain necessary and sufficient conditions on a SMOP $\left\{Q_{n}\right\}_{0}^{\infty}$ in order that a convex linear combination $\left\{R_{n}\right\}_{0}^{\infty}$ with $R_{n}=\beta \Phi_{n}+(1-\beta) Q_{n}$ be a SMOP with respect to a positive measure supported on the unit circle.
\end{abstract}

\section{INTRODUCTION}

Let $\mu$ be a finite positive Borel measure supported on $[0,2 \pi]$, and let $\left\{\varphi_{n}\right\}_{0}^{\infty}$ be the corresponding sequence of orthonormal polynomials, i.e.,

$$
\int_{0}^{2 \pi} \varphi_{n}\left(e^{i \theta}\right) \overline{\varphi_{m}\left(e^{i \theta}\right)} d \mu(\theta)=\delta_{n, m}, \quad n, m \geq 0,
$$

where $\varphi_{n}(z)=k_{n} z^{n}+$ lower degree terms, $k_{n}>0$.

We denote the sequence of monic orthogonal polynomials (SMOP) associated with $\mu$ by $\left\{\Phi_{n}\right\}_{0}^{\infty}$, where $\Phi_{n}=k_{n}{ }^{-1} \varphi_{n}$. It is well known that $\left\{\Phi_{n}\right\}_{0}^{\infty}$ satisfies for $n \geq 1$ the following recurrence relations:

$$
\begin{gathered}
\Phi_{n}(z)=z \Phi_{n-1}(z)+\Phi_{n}(0) \Phi_{n-1}^{*}(z), \\
\Phi_{n}^{*}(z)=\Phi_{n-1}^{*}(z)+\overline{\Phi_{n}(0)} z \Phi_{n-1}(z), \\
\Phi_{n}(z)=\left(1-\left|\Phi_{n}(0)\right|^{2}\right) z \Phi_{n-1}(z)+\Phi_{n}(0) \Phi_{n}^{*}(z), \\
\Phi_{n}^{*}(z)=\left(1-\left|\Phi_{n}(0)\right|^{2}\right) \Phi_{n-1}^{*}(z)+\overline{\Phi_{n}(0)} \Phi_{n}(z),
\end{gathered}
$$

where $\Phi_{n}^{*}(z)=z^{n} \overline{\Phi_{n}}\left(z^{-1}\right)$ is the reversed polynomial of $\Phi_{n}(z)$. For details about such recurrence relations, see [4], [5], and [9].

Furthermore, $\left\{\Phi_{n}\right\}_{0}^{\infty}$ satisfies the three-term recurrence relation

$$
\Phi_{n}(0) \Phi_{n+1}(z)=\left(z \Phi_{n}(0)+\Phi_{n+1}(0)\right) \Phi_{n}(z)-z\left(1-\left|\Phi_{n}(0)\right|^{2}\right) \Phi_{n+1}(0) \Phi_{n-1}(z),
$$

$n \geq 0$, with initial conditions $\Phi_{-1}(z)=0$ and $\Phi_{0}(z)=1$.

The values $\Phi_{n}(0)$ are called reflection parameters, and they satisfy $\left|\Phi_{n}(0)\right|<1$

Received by the editors March 4, 1996 and, in revised form, January 13, 1997.

1991 Mathematics Subject Classification. Primary 42C05.

Key words and phrases. Orthogonal polynomials, C-functions, measures on the unit circle.

The work of the first author was supported by the DGICYT under grant number PB93-1169.

The work of the second author was supported by an Acción Integrada Hispano-Austriaca 4B/1995. 
for $n \geq 1$. Conversely, given a sequence of complex numbers $a_{n}$ such that $\left|a_{n}\right|<1$, there exists a unique positive measure $\mu$ such that $a_{n}=\Phi_{n}(0)$, where $\left\{\Phi_{n}\right\}_{0}^{\infty}$ denotes the SMOP with respect to $\mu$. In a certain sense, this is the analogue of Favard's theorem on the unit circle (see [3]).

On the other hand, if we consider the second order linear difference equation

$$
a_{n} y_{n+1}=\left(z a_{n}+a_{n+1}\right) y_{n}-z\left(1-\left|a_{n}\right|^{2}\right) a_{n+1} y_{n-1}, \quad n \geq 1,
$$

the linear space of the solutions is two dimensional. Of course, one of the solutions is our SMOP $\left\{\Phi_{n}\right\}_{0}^{\infty}$, which corresponds to the initial conditions $y_{0}=1$ and $y_{1}=$ $z+a_{1}$.

If $a_{N}=0$ for some $N \geq 1$, then

$$
a_{N-1} y_{N}=z a_{N-1} y_{N-1} \quad \text { and } \quad a_{N+1} y_{N}=z a_{N+1} y_{N-1} .
$$

By convention $y_{N}=z y_{N-1}$ and $y_{N}(0)=a_{N}$. Furthermore, if $a_{N+1} \neq 0$, then $y_{N+2}$ can be given explicitly in terms of $y_{N}$ and $y_{N+1}$ by

$$
a_{N+1} y_{N+2}=\left(z a_{N+1}+a_{N+2}\right) y_{N+1}-z\left(1-\left|a_{N+1}\right|^{2}\right) a_{N+2} y_{N} .
$$

In such a case, we can choose $y_{N+1}=z y_{N}+a_{N+1} y_{N}=z^{2} y_{N-1}+a_{N+1} y_{N-1}$, because $a_{N+1} y_{N+2}(0)=a_{N+2} y_{N+1}(0)$. If $a_{N+1}=0$, again $y_{N+1}=z y_{N}$ and $y_{N+2}$ cannot be explicitly defined from the recurrence relation. If $a_{1} \neq 0$, a second linearly independent solution $\left\{\Psi_{n}\right\}_{0}^{\infty}$ of the above difference equation appears when $y_{0}=1$ and $y_{1}=z-a_{1}$. This means that $\Psi_{n}(0)=-a_{n}$ and, as a consequence, $\left\{\Psi_{n}\right\}_{0}^{\infty}$ is a SMOP explicitly given in terms of $\left\{\Phi_{n}\right\}_{0}^{\infty}$ in the following way:

$$
\Psi_{n}(z)=\frac{1}{c_{0}} \int_{0}^{2 \pi} \frac{e^{i \theta}+z}{e^{i \theta}-z}\left(\Phi_{n}\left(e^{i \theta}\right)-\Phi_{n}(z)\right) d \mu(\theta), \quad n \geq 1, \quad \Psi_{0}(z)=1,
$$

where $c_{0}=\int_{0}^{2 \pi} d \mu(\theta)$. These polynomials $\left\{\Psi_{n}\right\}_{0}^{\infty}$ are called polynomials of the second kind with respect to $\mu$.

Since $\left\{\Phi_{n}\right\}_{0}^{\infty}$ and $\left\{\Psi_{n}\right\}_{0}^{\infty}$ constitute a basis in the above linear space, it is straightforward to deduce that for $|\lambda|=1$ the SMOP $\left\{\Phi_{n}^{\lambda}\right\}_{0}^{\infty}$, whose reflection parameters are $\tilde{a}_{n}=\lambda a_{n}$ for $n \geq 1$, can be expressed in terms of $\left\{\Phi_{n}\right\}_{0}^{\infty}$ and $\left\{\Psi_{n}\right\}_{0}^{\infty}$ in the following way:

$$
\Phi_{n}^{\lambda}(z)=\frac{1+\lambda}{2} \Phi_{n}(z)+\frac{1-\lambda}{2} \Psi_{n}(z),
$$

i.e., a linear convex combination of the elements of the above basis (see [5, Section 7]).

The aim of this paper is to extend this property of convexity in order to characterize sequences of monic orthogonal polynomials $\left\{\Phi_{n}\right\}_{0}^{\infty}$ such that there exist $\beta \in \mathbb{C}$ and a SMOP $\left\{Q_{n}\right\}_{0}^{\infty}$ for which the sequence $\left\{R_{n}\right\}_{0}^{\infty}$ defined by

$$
R_{n}=\beta \Phi_{n}+(1-\beta) Q_{n}, \quad n \geq 0,
$$

is a SMOP. We will use the Carathéodory function (or C-function) associated with the measure $\mu$ defined by

$$
F(z)=\frac{1}{c_{0}} \int_{0}^{2 \pi} \frac{e^{i \theta}+z}{e^{i \theta}-z} d \mu(\theta)
$$

in order to obtain the C-function associated with $\left\{R_{n}\right\}_{0}^{\infty}$ in terms of $F$. Then the measure associated with $\left\{R_{n}\right\}_{0}^{\infty}$ can be easily deduced. 
In [2] the authors considered the problem of finding necessary and sufficient conditions on a SMOP $\left\{\Phi_{n}\right\}_{0}^{\infty}$ and a sequence of complex numbers $\left\{\alpha_{n}\right\}_{0}^{\infty}$ so that $\left\{\Phi_{n}+\alpha_{n} \Phi_{n-1}\right\}_{0}^{\infty}$ would be a SMOP. This result was extended in [6], where for a fixed $k$ a finite linear combination

$$
\Omega_{n}=\Phi_{n}+\sum_{j=n-k}^{n-1} \lambda_{n, j} \Phi_{j}
$$

is considered. $\left\{\Omega_{n}\right\}_{0}^{\infty}$ belongs to the Bernstein-Szegö class (see [9, Theorem 11.2, p. 289]), and $\left\{\Phi_{n}\right\}_{0}^{\infty}$ is a SMOP relative to a positive trigonometric rational weight function.

\section{MAin RESUlts}

Lemma 1. Let $\left\{\Phi_{n}\right\}_{0}^{\infty}$ and $\left\{\Psi_{n}\right\}_{0}^{\infty}$ be the SMOPs defined in (1.1) and (1.5) respectively and $\beta \in \mathbb{C}$.

i) If $|2 \beta-1|=1$, then the sequence $\left\{R_{n}\right\}_{0}^{\infty}$ given by

$$
R_{n}=\beta \Phi_{n}+(1-\beta) \Psi_{n}
$$

is always a SMOP.

ii) If $|2 \beta-1| \neq 1,(2.1)$ is a SMOP if and only if

$$
\Phi_{n}(z)=z^{n-1}(z+a) \text { for } n \geq 1 \text {, with }|a|<\min \left\{\frac{1}{|2 \beta-1|}, 1\right\} \text {, }
$$

or

$$
\Phi_{n}(z)=z^{n} \quad 1 \leq n \leq N \quad \text { and } \quad \Phi_{n}(z)=z^{n-(N+1)}\left(z^{N+1}+b\right) \quad n \geq N+1,
$$

for some $N \geq 1$, with $0<|b|<\min \{1 /|2 \beta-1|, 1\}$, i.e., $\left\{\Phi_{n}\right\}_{0}^{\infty}$ belongs to the Bernstein-Szegő class.

Proof. $\Rightarrow$ ) Because of (1.1), for $n \geq 0$

$$
R_{n+1}(z)=z R_{n}(z)+R_{n+1}(0) R_{n}^{*}(z) .
$$

Taking into account the definition of $R_{n}(z)$, we have for $n \geq 0$

$$
\begin{aligned}
& \beta \Phi_{n+1}(z)+(1-\beta) \Psi_{n+1}(z) \\
& \quad=\beta z \Phi_{n}(z)+(1-\beta) z \Psi_{n}(z)+R_{n+1}(0)\left(\bar{\beta} \Phi_{n}^{*}(z)+(1-\bar{\beta}) \Psi_{n}^{*}(z)\right) .
\end{aligned}
$$

Thus,

$$
\beta \Phi_{n+1}(0) \Phi_{n}^{*}-(1-\beta) \Phi_{n+1}(0) \Psi_{n}^{*}=R_{n+1}(0)\left(\bar{\beta} \Phi_{n}^{*}+(1-\bar{\beta}) \Psi_{n}^{*}\right),
$$

or, equivalently,

$$
\left(\beta \Phi_{n+1}(0)-\bar{\beta} R_{n+1}(0)\right) \Phi_{n}^{*}=\left((1-\bar{\beta}) R_{n+1}(0)+(1-\beta) \Phi_{n+1}(0)\right) \Psi_{n}^{*} .
$$

Using

$$
R_{n}(0)=\beta \Phi_{n}(0)-(1-\beta) \Phi_{n}(0)=(2 \beta-1) \Phi_{n}(0)
$$

in the above relation, we obtain for $n \geq 0$

$$
(\beta-\bar{\beta}(2 \beta-1)) \Phi_{n+1}(0) \Phi_{n}^{*}=((1-\bar{\beta})(2 \beta-1)+(1-\beta)) \Phi_{n+1}(0) \Psi_{n}^{*} .
$$

That is,

$$
\left(\beta+\bar{\beta}-2|\beta|^{2}\right) \Phi_{n+1}(0) \Phi_{n}^{*}=\left(\beta+\bar{\beta}-2|\beta|^{2}\right) \Phi_{n+1}(0) \Psi_{n}^{*},
$$


from which it follows that

$$
\left(1-|1-2 \beta|^{2}\right) \Phi_{n+1}(0)\left(\Phi_{n}^{*}-\Psi_{n}^{*}\right)=0,
$$

or, equivalently,

$$
\left(1-|1-2 \beta|^{2}\right) \overline{\Phi_{n+1}(0)}\left(\Phi_{n}-\Psi_{n}\right)=0, \quad n \geq 0 .
$$

Therefore, either $|1-2 \beta|=1$ or, if $|1-2 \beta| \neq 1$, then $\overline{\Phi_{n+1}(0)}\left(\Phi_{n}-\Psi_{n}\right)=0$ for $n \geq$ 0 . In this second case, either $\Phi_{n+1}(0)=0$ for every $n \geq 0$, or there exists at most one $\Phi_{N}$ with $N \geq 1$ such that $\Phi_{N}(0) \neq 0$. To prove this, suppose $\Phi_{N}(0) \neq 0$ and $\Phi_{M}(0) \neq 0$ with $M>N \geq 1$. Then $\Phi_{M-1}=\Psi_{M-1}$, i.e., $\Phi_{M-1}(0)=0$. Thus, if we use the recurrence relation (1.1), then $\Phi_{M-2}=\Psi_{M-2}$. After a finite number of steps, we get $\Phi_{N}=\Psi_{N}$, i.e., $\Phi_{N}(0)=0$, and this yields a contradiction.

We conclude that

either $\Phi_{n+1}(0)=0$ for $n \geq 1$, i.e., $\Phi_{n}(z)=z^{n-1}(z+a)$ for $n \geq 1$, where $|a|<1$, or there exists a unique $N \geq 1$ such that $\Phi_{N+1}(0) \neq 0$ and $\Phi_{n}(0)=0$ for $n \neq N+1$. Then $\Phi_{n}(z)=z^{n-(N+1)} \Phi_{N+1}(z)$ for $n \geq N+1$, and $\Phi_{n}(z)=z^{n}$ for $1 \leq n \leq N$.

$\Leftarrow)$ If $|2 \beta-1|=1$, then $2 \beta=1+\lambda$ with $|\lambda|=1$. Thus $\beta=\frac{1+\lambda}{2}$ and $R_{n}(z)=\Phi_{n}^{\lambda}(z)$ follows from (1.6).

If $|2 \beta-1| \neq 1$ and $\Phi_{n}(z)=z^{n-(N+1)} \Phi_{N+1}(z)$ for $n \geq N+1$ and $\Phi_{n}(z)=z^{n}$ for $1 \leq n \leq N$, it is easy to check that

$$
R_{n+1}(z)=\beta \Phi_{n+1}(z)+(1-\beta) \Psi_{n+1}(z)=z\left(\beta \Phi_{n}(z)+(1-\beta) \Psi_{n}(z)\right)=z R_{n}(z)
$$

when $n \geq N+1$ or $n<N$. Furthermore, $R_{N+1}(0)=(2 \beta-1) \Phi_{N+1}(0)$, and since $\left|\Phi_{N+1}(0)\right|<\min \left\{\frac{1}{|2 \beta-1|}, 1\right\}$, then $\left|R_{N+1}(0)\right|<1$. If $\Phi_{n}(z)=z^{n-1} \Phi_{1}(z)$ for $n \geq$ 1 , then $R_{n+1}(z)=z R_{n}(z), n \geq 1$, and since $\left|\Phi_{1}(0)\right|<\min \left\{\frac{1}{|2 \beta-1|}, 1\right\}$, then $\left|R_{1}(0)\right|<1$. Therefore $\left\{R_{n}\right\}_{0}^{\infty}$ is a SMOP.

Remark 1 . Notice that from ii) in the above lemma, we can obtain the sequence $\left\{R_{n}\right\}_{0}^{\infty}$ as follows:

- If $\Phi_{n}(z)=z^{n-1} \Phi_{1}(z)=z^{n-1}(z+a), \quad n \geq 1$, then

$$
R_{n}(z)=\beta z^{n-1}(z+a)+(1-\beta) z^{n-1}(z-a)=z^{n-1}(z+(2 \beta-1) a), \quad n \geq 1 \text {. }
$$

- If $\Phi_{1}(0)=0$ and $\Phi_{N+1}(0) \neq 0$ for some $N \geq 1$, then

$$
R_{n}(z)=z^{n}, \quad n \leq N
$$

and

$$
\begin{aligned}
R_{n}(z) & =\beta z^{n-1}\left(z+\Phi_{N+1}(0) z^{-N}\right)+(1-\beta) z^{n-1}\left(z-\Phi_{N+1}(0) z^{-N}\right) \\
& =z^{n-1}\left(z+(2 \beta-1) \Phi_{N+1}(0) z^{-N}\right), \quad n \geq N+1 .
\end{aligned}
$$

Lemma 2. Let $\left\{\Phi_{n}\right\}_{0}^{\infty}$ be a SMOP. For $\lambda \neq 1$, let $\left\{\Phi_{n}^{\lambda}\right\}_{0}^{\infty}$ be as in (1.6). Then the sequence $\left\{R_{n}\right\}_{0}^{\infty}$ given by

$$
R_{n}=\beta \Phi_{n}+(1-\beta) \Phi_{n}^{\lambda}
$$

is a SMOP if and only if either

i) $\left|\beta+\frac{\lambda}{1-\lambda}\right|=\frac{1}{|1-\lambda|}$, or 
ii) $\left|\beta+\frac{\lambda}{1-\lambda}\right| \neq \frac{1}{|1-\lambda|}$, and $\left\{\Phi_{n}\right\}_{0}^{\infty}$ is either of the form

$$
\Phi_{n}(z)=z^{n-1}(z+a) \quad \text { for } n \geq 1, \text { with } \quad|a|<\min \left\{\frac{1}{|(1-\lambda) \beta+\lambda|}, 1\right\},
$$

or of the form

$\Phi_{n}(z)=z^{n}, \quad 1 \leq n \leq N, \quad$ and $\quad \Phi_{n}(z)=z^{n-(N+1)}\left(z^{N+1}+b\right), \quad n \geq N+1$, for some $N \geq 1$, with $0<|b|<\min \{1 /|(1-\lambda) \beta+\lambda|, 1\}$.

Proof. By (1.6), (2.2) can be reduced to

$$
\begin{aligned}
R_{n} & =\beta \Phi_{n}+(1-\beta)\left(\frac{1+\lambda}{2} \Phi_{n}+\frac{1-\lambda}{2} \Psi_{n}\right) \\
& =\left(\beta+(1-\beta) \frac{1+\lambda}{2}\right) \Phi_{n}+\frac{(1-\beta)(1-\lambda)}{2} \Psi_{n} .
\end{aligned}
$$

According to Lemma $1,\left\{R_{n}\right\}_{0}^{\infty}$ is a SMOP if and only if either

i) $|2 \beta+(1-\beta)(1+\lambda)-1|=|(1-\lambda) \beta+\lambda|=1$,

or

ii) $|(1-\lambda) \beta+\lambda| \neq 1$, in which case $\left\{\Phi_{n}\right\}_{0}^{\infty}$ is the corresponding SMOP defined as in Lemma 1, ii).

Theorem 1. Let $\left\{\Phi_{n}\right\}_{0}^{\infty}$ and $\left\{Q_{n}\right\}_{0}^{\infty}$ be two SMOPs. Let

$$
R_{n}=\beta \Phi_{n}+(1-\beta) Q_{n}, \quad n \geq 0,
$$

where $\beta \in \mathbb{C} \backslash\{0,1\}$. Then $\left\{R_{n}\right\}_{0}^{\infty}$ is a SMOP if and only if

$$
Q_{n}(0)=\Phi_{n}(0) \text { for } n \leq N, Q_{n}(0)=\Phi_{n}^{\lambda}(0) \text { for } n \geq N+2 \text {, with } N \geq 0
$$

and either

i) $Q_{N+1}(0)=\Phi_{N+1}^{\lambda}(0)$

or

ii) $Q_{N+1}(0) \neq \Phi_{N+1}^{\lambda}(0)$, and $\left|\beta \Phi_{N+1}(0)+(1-\beta) Q_{N+1}(0)\right|<1$, with $\lambda=$ $\beta(1-\bar{\beta}) / \bar{\beta}(1-\beta)$.

Proof. $\Rightarrow$ ) Let us suppose that $\left\{R_{n}\right\}_{0}^{\infty}$ is a SMOP. Then

$$
\begin{aligned}
& \beta \Phi_{n+1}(z)+(1-\beta) Q_{n+1}(z) \\
& \quad=z\left(\beta \Phi_{n}(z)+(1-\beta) Q_{n}(z)\right)+R_{n+1}(0)\left(\bar{\beta} \Phi_{n}^{*}(z)+(1-\bar{\beta}) Q_{n}^{*}(z)\right) .
\end{aligned}
$$

Using the recurrence relations for $\left\{\Phi_{n}\right\}_{0}^{\infty}$ and $\left\{Q_{n}\right\}_{0}^{\infty}$, we have

$$
\left(\beta \Phi_{n+1}(0)-\bar{\beta} R_{n+1}(0)\right) \Phi_{n}^{*}(z)=\left((1-\bar{\beta}) R_{n+1}(0)-(1-\beta) Q_{n+1}(0)\right) Q_{n}^{*}(z) .
$$

Thus

$$
\begin{aligned}
& \left(\beta(1-\bar{\beta}) \Phi_{n+1}(0)-\bar{\beta}(1-\beta) Q_{n+1}(0)\right) \Phi_{n}^{*}(z) \\
= & \left(\beta(1-\bar{\beta}) \Phi_{n+1}(0)-\bar{\beta}(1-\beta) Q_{n+1}(0)\right) Q_{n}^{*}(z)
\end{aligned}
$$

or, equivalently,

$\left(\bar{\beta}(1-\beta) \overline{\Phi_{n+1}(0)}-\beta(1-\bar{\beta}) \overline{Q_{n+1}(0)}\right)\left(\Phi_{n}(z)-Q_{n}(z)\right)=0, \quad$ for $\quad n \geq 0$.

Let $A=\left\{n \geq 0: \bar{\beta}(1-\beta) \overline{\Phi_{n+1}(0)} \neq \beta(1-\bar{\beta}) \overline{Q_{n+1}(0)}\right\}$.

1 ) If $A$ is a finite set, we will consider two situations: 
1.i) $A=\varnothing$ leads to $Q_{n+1}(0)=\frac{\beta(1-\bar{\beta})}{\bar{\beta}(1-\beta)} \Phi_{n+1}(0)$, that is,

$$
Q_{n+1}(0)=\lambda \Phi_{n+1}(0) \quad \text { for } \quad n \geq 0 \quad \text { and } \quad \lambda=\frac{\beta(1-\bar{\beta})}{\bar{\beta}(1-\beta)} .
$$

1.ii) If $A \neq \varnothing$, let $M=\max A$. Then $\Phi_{M}=Q_{M}$ and as a consequence of the recurrence relation (1.3) we have $Q_{n}=\Phi_{n}$ for $n \leq M$.

On the other hand, since

$$
\beta(1-\bar{\beta}) \Phi_{n+1}(0)=\bar{\beta}(1-\beta) Q_{n+1}(0) \quad \text { for } \quad n>M
$$

then

$$
Q_{n+1}(0)=\lambda \Phi_{n+1}(0), \quad \text { where } \quad \lambda=\frac{\beta(1-\bar{\beta})}{\bar{\beta}(1-\beta)}, \quad \text { for } \quad n \geq M+1 .
$$

2) If $A$ is an infinite set, given $N \geq 0$ there exists $M^{\prime} \in A$ such that $M^{\prime}>N$. Then, as before, $\Phi_{M^{\prime}}=Q_{M^{\prime}}$ and $Q_{n}=\Phi_{n}$ for $n \leq M^{\prime}$, i.e., $Q_{n}=\Phi_{n}$ for $n \geq 0$.

$\Leftarrow)$ Straightforward calculations give

$$
\begin{aligned}
R_{n+1}(z) & -z R_{n}(z)-R_{n+1}(0) R_{n}^{*}(z) \\
= & \left(\beta \Phi_{n+1}(0)-\bar{\beta} R_{n+1}(0)\right) \Phi_{n}^{*}(z) \\
& +\left((1-\beta) Q_{n+1}(0)-(1-\bar{\beta}) R_{n+1}(0)\right) Q_{n}^{*}(z) \\
= & \left(\beta(1-\bar{\beta}) \Phi_{n+1}(0)-\bar{\beta}(1-\beta) Q_{n+1}(0)\right) \Phi_{n}^{*}(z) \\
& -\left(\beta(1-\bar{\beta}) \Phi_{n+1}(0)-\bar{\beta}(1-\beta) Q_{n+1}(0)\right) Q_{n}^{*}(z) \\
= & \left(\beta(1-\bar{\beta}) \Phi_{n+1}(0)-\bar{\beta}(1-\beta) Q_{n+1}(0)\right)\left(\Phi_{n}^{*}(z)-Q_{n}^{*}(z)\right) .
\end{aligned}
$$

If $Q_{n}(0)=\Phi_{n}(0)$ for $n \leq N$, then $Q_{n}=\Phi_{n}$ for $n \leq N$ and the above expression vanishes for $n \leq N$. If $Q_{n}(0)=\Phi_{n}^{\lambda}(0)$ for $n \geq N+2$, the above expression vanishes for $n \geq N+1$.

Remark 2. 1) If $\beta \in \mathbb{R} \backslash\{0,1\}$, then $\lambda=1$ and $Q_{n}(0)=\Phi_{n}(0)$ for $n \neq N+1$. This is a perturbation of the reflection parameter $\Phi_{N+1}(0)$, while the others remain invariant. If $Q_{N+1}(0) \neq \Phi_{N+1}(0)$, then $R_{n}(0)=\Phi_{n}(0)$ for $n \neq N+1$, and $R_{N+1}(0)$ $=\Phi_{N+1}(0)+\alpha$.

On the other hand, $R_{n}(z)=\Phi_{n}(z)$ for $n \leq N$, and $R_{N+1}(z)=\Phi_{N+1}(z)+$ $\alpha \Phi_{n}^{*}(z)$. For the other terms of the sequence $\left\{R_{n}\right\}_{0}^{\infty}$, notice that

$$
R_{n}^{(N+1)}(z)=\Phi_{n}^{(N+1)}(z), \quad n \geq 0,
$$

where the superscript denotes the $(N+1)$ th associated polynomial introduced in [7, Definition 3.1]. But according to [7, Theorem 3.1], we have for $n \geq 0$

$$
\begin{aligned}
& \Phi_{n}^{(N+1)}(z) \\
& \quad=\frac{\left(\Psi_{N+1}(z)+\Psi_{N+1}^{*}(z)\right) \Phi_{n+N+1}(z)+\left(\Phi_{N+1}^{*}(z)-\Phi_{N+1}(z)\right) \Psi_{n+N+1}(z)}{d_{N+1} z^{N+1}}, \\
& \Psi_{n}^{(N+1)}(z) \\
& \quad=\frac{\left(\Phi_{N+1}(z)+\Phi_{N+1}^{*}(z)\right) \Psi_{n+N+1}(z)+\left(\Psi_{N+1}^{*}(z)-\Psi_{N+1}(z)\right) \Phi_{n+N+1}(z)}{d_{N+1} z^{N+1}},
\end{aligned}
$$


where

$$
d_{N+1}=2 c_{0} \prod_{k=1}^{N+1}\left(1-\left|\Phi_{k}(0)\right|^{2}\right) .
$$

Then, if $\left\{S_{n}\right\}_{0}^{\infty}$ denotes the SMOP of second kind associated with $\left\{R_{n}\right\}_{0}^{\infty}$,

$$
\begin{aligned}
\left(S_{N+1}+S_{N+1}^{*}\right) R_{n+N+1}+\left(R_{N+1}^{*}-\right. & \left.R_{N+1}\right) S_{n+N+1} \\
=\frac{\left(1-\left|\Phi_{N+1}(0)+\alpha\right|^{2}\right)}{1-\left|\Phi_{N+1}(0)\right|^{2}}\left(\left(\Psi_{N+1}\right.\right. & \left.+\Psi_{N+1}^{*}\right) \Phi_{n+N+1} \\
& \left.+\left(\Phi_{N+1}^{*}-\Phi_{N+1}\right) \Psi_{n+N+1}\right),
\end{aligned}
$$

and

$$
\begin{aligned}
\left(R_{N+1}+R_{N+1}^{*}\right) S_{n+N+1}+\left(S_{N+1}^{*}-\right. & \left.S_{N+1}\right) R_{n+N+1} \\
=\frac{\left(1-\left|\Phi_{N+1}(0)+\alpha\right|^{2}\right)}{1-\left|\Phi_{N+1}(0)\right|^{2}}\left(\left(\Phi_{N+1}\right.\right. & \left.+\Phi_{N+1}^{*}\right) \Psi_{n+N+1} \\
& \left.+\left(\Psi_{N+1}^{*}-\Psi_{N+1}\right) \Phi_{n+N+1}\right) .
\end{aligned}
$$

Denoting

$$
R_{ \pm}=R_{N+1} \pm R_{N+1}^{*}=\left(\Phi_{N+1} \pm \Phi_{N+1}^{*}\right)+\left(\alpha \Phi_{N}^{*} \pm \bar{\alpha} z \Phi_{N}\right)
$$

and

$$
S_{ \pm}=S_{N+1} \pm S_{N+1}^{*}=\left(\Psi_{N+1} \pm \Psi_{N+1}^{*}\right)-\left(\alpha \Psi_{N}^{*} \pm \bar{\alpha} z \Psi_{N}\right),
$$

formulas (2.3) and (2.4) may be expressed in matrix form as follows:

$$
\left(\begin{array}{cc}
S_{+} & -R_{-} \\
-S_{-} & R_{+}
\end{array}\right)\left(\begin{array}{c}
R_{n+N+1} \\
S_{n+N+1}
\end{array}\right)=\alpha_{N}\left(\begin{array}{cc}
\Psi_{+} & -\Phi_{-} \\
-\Psi_{-} & \Phi_{+}
\end{array}\right)\left(\begin{array}{l}
\Phi_{n+N+1} \\
\Psi_{n+N+1}
\end{array}\right) .
$$

Then

$$
\begin{gathered}
\left(\begin{array}{c}
R_{n+N+1} \\
S_{n+N+1}
\end{array}\right)=\alpha_{N}\left(\begin{array}{cc}
S_{+} & -R_{-} \\
-S_{-} & R_{+}
\end{array}\right)^{-1}\left(\begin{array}{cc}
\Psi_{+} & -\Phi_{-} \\
-\Psi_{-} & \Phi_{+}
\end{array}\right)\left(\begin{array}{l}
\Phi_{n+N+1} \\
\Psi_{n+N+1}
\end{array}\right) \\
=\frac{\alpha_{N}}{S_{+} R_{+}-S_{-} R_{-}}\left(\begin{array}{cc}
R_{+} & R_{-} \\
S_{-} & S_{+}
\end{array}\right)\left(\begin{array}{cc}
\Psi_{+} & -\Phi_{-} \\
-\Psi_{-} & \Phi_{+}
\end{array}\right)\left(\begin{array}{l}
\Phi_{n+N+1} \\
\Psi_{n+N+1}
\end{array}\right) .
\end{gathered}
$$

Therefore,

$$
\begin{aligned}
R_{n+N+1}=\frac{\alpha_{N}}{S_{+} R_{+}-S_{-} R_{-}}\left(\left(R_{+} \Psi_{+}\right.\right. & \left.-R_{-} \Psi_{-}\right) \Phi_{n+N+1} \\
& \left.-\left(R_{+} \Phi_{-}-R_{-} \Phi_{+}\right) \Psi_{n+N+1}\right)
\end{aligned}
$$

and

$$
\begin{aligned}
S_{n+N+1}=\frac{\alpha_{N}}{S_{+} R_{+}-S_{-} R_{-}}\left(\left(S_{-} \Psi_{+}\right.\right. & \left.-S_{+} \Psi_{-}\right) \Phi_{n+N+1} \\
& \left.+\left(S_{+} \Phi_{+}-S_{-} \Phi_{-}\right) \Psi_{n+N+1}\right) .
\end{aligned}
$$

Thus, the relation between the corresponding C-functions is

$$
\tilde{F}=\frac{A+B F}{C+D F},
$$

where $A=S_{+} \Psi_{-}-S_{-} \Psi_{+}, B=\Phi_{+} S_{+}-S_{-} \Phi_{-}, C=R_{+} \Psi_{+}-R_{-} \Psi_{-}$and $D=$ $\Phi_{-} R_{+}-R_{-} \Phi_{+}$are self-reciprocal polynomials. 
Hence, as in [7, Theorem 2.3] we can obtain the measure $\tilde{\mu}$ associated with $\tilde{F}$. by

2) If $\beta \in \mathbb{C} \backslash \mathbb{R}$, then $\left\{Q_{n}(0)\right\}_{0}^{\infty}$ is a perturbation of the sequence $\left\{\Phi_{n}^{\lambda}(0)\right\}_{0}^{\infty}$ given

$$
Q_{n}(0)=\Phi_{n}(0), \quad n \leq N, \quad \text { and } \quad Q_{N+1}(0) \neq \Phi_{N+1}^{\lambda}(0)
$$

for some $N \geq 0$. Using arguments similar to those employed above (taking into account that $\left.Q_{n}^{(N+1)}(z)=\left(\Phi_{n}^{\lambda}\right)^{(N+1)}(z)\right)$, we obtain a relation between the corresponding C-functions analogous to (2.5).

3) For both cases

$$
R_{n}(0)=\Phi_{n}(0), \quad \text { for } \quad n \leq N
$$

and

$$
\begin{aligned}
R_{n}(0) & =\beta \Phi_{n}(0)+(1-\beta) \Phi_{n}^{\lambda}(0)=(\beta+\lambda(1-\beta)) \Phi_{n}(0) \\
& =\left(\beta+\frac{\beta(1-\bar{\beta})}{\bar{\beta}}\right) \Phi_{n}(0)=\frac{\beta}{\bar{\beta}} \Phi_{n}(0)=\mu \Phi_{n}(0),
\end{aligned}
$$

for $n \geq N+2$, with $|\mu|=1$.

Thus, we have proved

Corollary 1. Under the assumptions of Theorem 1, the sequence $\left\{R_{n}\right\}_{0}^{\infty}$ given by

$$
R_{n}=\beta \Phi_{n}+(1-\beta) Q_{n}
$$

is a SMOP if and only

$$
R_{n}=\Phi_{n}, \quad n \leq N, \text { and } R_{n}(0)=\Phi_{n}^{\mu}(0)=\mu \Phi_{n}(0), \quad n \geq N+2,
$$

with $N \geq 0$ and $\mu=\beta / \bar{\beta}$.

Thus, $\left\{R_{n}\right\}_{0}^{\infty}$ is a finite perturbation of $\left\{\Phi_{n}^{\mu}\right\}_{0}^{\infty}$, and the first $N+1$ reflection parameters are given by $\left\{\Phi_{n}(0)\right\}_{0}^{N}$ with the convention that $\Phi_{0}(0)=1$.

Such perturbations were introduced in [7]. For more details, see [8]. Notice that the C-function associated with $\left\{\Phi_{n}^{\mu}\right\}_{0}^{\infty}$ (see [1]) is

$$
F^{\mu}=\frac{(\mu-1)+(\mu+1) F}{(\mu+1)+(1-\mu) F} \text {. }
$$

\section{ACKNOWLEDGEMENTS}

We are grateful to Professor Guillermo López Lagomasino for his very helpful comments on the revised version of this manuscript.

\section{REFERENCES}

1. M. Alfaro, M. P. Alfaro, J. Guadalupe, and L. Vigil, Correspondance entre suites de polynômes orthogonaux et fonctions de la boule unité de $H_{0}^{\infty}(D)$, Lect. Notes in Math., vol 1171, Springer Verlag, Berlin (1985), 158-163. MR 87h:30073

2. A. Branquinho, L. B. Golinskii, and F. Marcellán, Rational modifications of Lebesgue measure on the unit circle and an inverse problem, submitted.

3. T. Erdélyi, J. S. Geronimo, P. Nevai, and J. Zhang, A simple proof of Favard's theorem on the unit circle, Atti. Sem. Mat. Fis. Univ. Modena 28 (1991), 41-46. MR 92m:42025

4. G. Freud, Orthogonal polynomials, Pergamon Press, Oxford 1971. MR 58:1982 (French original)

5. Ya L. Geronimus, Polynomials Orthogonal on a circle and their applications, Amer. Math. Soc. Transl. (1), vol. 3, Providence, Rhode Island (1962), 1-78. MR 15:869i 
6. F. Marcellán, F. Peherstorfer, and R. Steinbauer, Orthogonality properties of linear combinations of orthogonal polynomials, Adv. in Comp. Math. 5 (1996), 281-295. CMP 97:02

7. F. Peherstorfer, A special class of polynomials orthogonal on the unit circle including the associated polynomials, Constr. Approx. 12 (1996), 161-186. MR 97d:42023

8. F. Peherstorfer and R. Steinbauer, Perturbation of orthogonal polynomials on the unit circle: A survey, Proceedings Workshop on orthogonal polynomials on the unit circle. M. Alfaro et al. Editors, Universidad Carlos III de Madrid (1994), 97-119. MR 95j:42022

9. G. Szegö, Orthogonal Polynomials, Amer. Math. Soc. Colloq. Publ. 23, Providence, Rhode Island 1975, 4th edition. MR 51:8724

Departamento de Matemática Aplicada, E.T.S.I.I., Universidad de Vigo, Spain

E-mail address: acachafe@dma.uvigo.es

Departamento de Matemáticas, E.P.S., Universidad Carlos III de Madrid, Spain

E-mail address: pacomarc@ing.uc3m.es 